ANNALES

POLONICI MATHEMATICI

$92.3(2007)$

\title{
Fite and Kamenev type oscillation criteria for second order elliptic equations
}

\author{
by Zhiting Xu (Guangzhou)
}

\begin{abstract}
Fite and Kamenev type oscillation criteria for the second order nonlinear damped elliptic differential equation

$$
\sum_{i, j=1}^{N} D_{i}\left[a_{i j}(x) D_{j} y\right]+\sum_{i=1}^{N} b_{i}(x) D_{i} y+p(x) f(y)=0
$$

are obtained. Our results are extensions of those for ordinary differential equations and improve some known oscillation criteria in the literature. Several examples are given to show the significance of the results.
\end{abstract}

1. Introduction. Consider the second order nonlinear damped elliptic differential equation

$$
\sum_{i, j=1}^{N} D_{i}\left[a_{i j}(x) D_{j} y\right]+\sum_{i=1}^{N} b_{i}(x) D_{i} y+p(x) f(y)=0
$$

in $\Omega(a)$, where $N \geq 2, x=\left(x_{1}, \ldots, x_{N}\right) \in \mathbb{R}^{N},|x|=\left[\sum_{i=1}^{N} x_{i}^{2}\right]^{1 / 2}, D_{i}=$ $\partial / \partial x_{i}$ for all $i$, and $\Omega(a)=\left\{x \in \mathbb{R}^{N}:|x| \geq a\right\}$ for some $a>0$.

Throughout this paper we shall assume that

(A1) $A=\left(a_{i j}\right)_{N \times N}$ is a real symmetric positive definite matrix function with $a_{i j} \in C_{\text {loc }}^{1+\nu}(\Omega(a), \mathbb{R})$ for all $i, j, \nu \in(0,1)$, and there exists a function $\lambda \in C^{1}\left([a, \infty), \mathbb{R}^{+}\right)$such that

$$
\lambda(r) \geq \max _{|x|=r} \lambda_{\max }(x) \quad \text { for } r \geq a,
$$

where $\lambda_{\max }(x)$ denotes the largest eigenvalue of the matrix $A$;

(A2) $B^{\mathrm{T}}=\left(b_{i}(x)\right)_{1 \times N}, b_{i} \in C_{\text {loc }}^{\nu}(\Omega(a), \mathbb{R})$ for all $i$, and $p \in C_{\text {loc }}^{\nu}(\Omega(a), \mathbb{R})$; (A3) $f \in C(\mathbb{R}, \mathbb{R}) \cup C^{1}(\mathbb{R}-\{0\}, \mathbb{R}), f^{\prime}(y) \geq k>0, y f(y)>0$ for $y \neq 0$.

2000 Mathematics Subject Classification: 35B05, 35J60, 34C10.

Key words and phrases: oscillation, second order, nonlinear, elliptic differential equation, damped. 
A function $y \in C_{\mathrm{loc}}^{2+\nu}(\Omega(a), \mathbb{R})$ is said to be a solution of $(1.1)$ in $\Omega(a)$ if $y(x)$ satisfies (1.1) for all $x \in \Omega(a)$. For existence of solutions of (1.1), we refer the reader to the monograph [5]. We restrict our attention to the nontrivial solutions $y(x)$ of (1.1), i.e. $\sup \{|y(x)|:|x|>b\}>0$ for any $b \geq a$. A nontrivial solution $y(x)$ of $(1.1)$ is called oscillatory if the set $\{x \in \Omega(a): y(x)=0\}$ is unbounded; otherwise it is said to be nonoscillatory. Equation (1.1) is oscillatory if all its solutions are oscillatory.

Equation (1.1) is a very important type of partial differential equations (PDE), for such equations have wide applications in physics, biology and glaciology (see [5]). In the qualitative theory of nonlinear PDE, one of the important themes is to determine whether or not solutions of the equation under consideration are oscillatory. In the last decades, there has been an increasing interest in obtaining sufficient conditions for the oscillation and/or nonoscillation of solutions for different classes of second order elliptic differential equations (see for example, $[1,2,7-14,16-22]$ and the references therein). In particular, for the semilinear elliptic differential equation

$$
\sum_{i, j=1}^{N} D_{i}\left[a_{i j}(x) D_{j} y\right]+p(x) f(y)=0
$$

in 1980, by using the $N$-dimensional vector partial Riccati transformation

$$
w(x)=-\frac{\alpha(|x|)}{f(y(x))}(A \nabla y)(x),
$$

where $\alpha \in C^{2}(0, \infty)$ is an arbitrary positive function and $\nabla y$ denotes the gradient of $y$, Noussair and Swanson [13] first extended the well-known Fite theorem [4] to (1.2). Very recently, the author [19] obtained Kamenev type theorems [6] for (1.2). Therefore, it is natural to ask if the results of Fite [4] and Kamenev [6] can be extended to the general equation (1.1). However, to the best of our knowledge, Fite and Kamenev type oscillation theorems have not been well developed for (1.1). This is because the Riccati substitution (1.3), which plays a key role in the proofs of the results for (1.2), is an $N$-dimensional vector function, which prevents simple extensions of existing work for ordinary differential equations.

In this paper, we will take the challenge of extending the work of Fite [4] and Kamenev [6] to (1.1). By applying an $N$-dimensional vector inequality (Lemma 2.1), and with a careful discussion of the Riccati inequality (Lemma 2.2), we will establish Fite and Kamenev type criteria for (1.1). The results obtained are quite general; with the appropriate choice of the functions $\eta$, $\phi$ and $H$, we can deduce a series of effective oscillation criteria for (1.2). Obviously, our results cover the main results in $[13,19]$ for $(1.2)$ as special cases and are new even for (1.2). Three examples will be given to show the significance of our main results. 
2. Fite type criteria. In this section we will establish generalized Fite type oscillation criteria for equation (1.1). Our approach is based largely on Coles's technique [3]. For simplicity, we use the following notations. For any given function $\eta \in C^{1}([a, \infty), \mathbb{R})$, define

$$
\begin{aligned}
\varrho(r) & =\exp \left(-\frac{k}{\omega_{N}} \int_{a}^{r} \eta(s) s^{1-N} d s\right), \\
g(r) & =\frac{2 \omega_{N}}{k} \lambda(r) \varrho(r) r^{N-1}, \\
p_{M}(r) & =\int_{S_{r}}\left(p(x)-\frac{1}{2 k} \lambda_{\max }(x)\left|B^{\mathrm{T}} A^{-1}\right|^{2}\right) d \sigma, \\
\theta(r) & =\varrho(r)\left\{p_{M}(r)+\frac{k}{2 \omega_{N}} r^{1-N} \lambda(r) \eta^{2}(r)-(\lambda(r) \eta(r))^{\prime}\right\},
\end{aligned}
$$

where $S_{r}=\left\{x \in \mathbb{R}^{N}:|x|=r\right\}$ for $r>0$, and $\omega_{N}$ and $d \sigma$ denote the surface measure of the unit sphere and the spherical integral element in $\mathbb{R}^{N}$, respectively.

Let $\Im$ denote the class of all positive and locally integrable, but not integrable functions. Let $\phi \in \Im$ and, for $b \geq a$, define

$$
\alpha(r, b)=\int_{b}^{r} \phi(s) d s, \quad \beta(r, b)=\frac{1}{\phi(r)} \int_{b}^{r} g(s) \phi^{2}(s) d s,
$$

and

$$
\Psi_{\phi}(r, b)=\frac{1}{\alpha(r, b)} \int_{b}^{r} \phi(s) \int_{b}^{s} \theta(u) d u d s .
$$

In order to establish our theorems, we need the following technical lemmas. The first is an $N$-dimensional vector inequality which plays a major role in dealing with the damping terms of (1.1). The second provides relations between positive solutions of (1.1) and of a 1-dimensional Riccati inequality.

Lemma 2.1. Let $u, v \in \mathbb{R}^{N}, c>0$. Then

$$
c u \cdot u^{\mathrm{T}}+u \cdot v^{\mathrm{T}} \geq \frac{c}{2} u \cdot u^{\mathrm{T}}-\frac{1}{2 c} v \cdot v^{\mathrm{T}} .
$$

The proof Lemma 2.1 is straightforward and omitted.

Lemma 2.2. Let $\eta \in C^{1}([a, \infty), \mathbb{R})$ and $y(x)$ be a positive solution of (1.1) in $\Omega\left(b_{0}\right)$. Put

$$
Z(r)=\varrho(r)\left[\int_{S_{r}} W(x) \cdot \nu(x) d \sigma+\lambda(r) \eta(r)\right] \quad \text { for } r \geq b_{0},
$$


where $W(x)=(A \nabla y)(x) / f(y(x)), \nu(x)=x /|x|,|x| \neq 0$, denotes the outward unit normal. Then $Z(r)$ satisfies the Riccati inequality

$$
Z^{\prime}(r) \leq-\theta(r)-\frac{1}{g(r)} Z^{2}(r), \quad r>b_{0} .
$$

Proof. Differentiation of the $i$ th component of $W(x)$ with respect to $x_{i}$ gives

$$
D_{i} W(x)_{i}=-\frac{f^{\prime}(y)}{f^{2}(y)} D_{i} y\left(\sum_{j=1}^{N} a_{i j}(x) D_{j} y\right)+\frac{1}{f(y)} D_{i}\left(\sum_{j=1}^{N} a_{i j}(x) D_{j} y\right)
$$

for all $i$. Summation over $i$ and application of (1.1) lead to

$$
\begin{aligned}
& \operatorname{div} W(x) \\
= & -f^{\prime}(y)\left(W^{\mathrm{T}} A^{-1} W\right)(x)-\left(B^{\mathrm{T}} A^{-1} W\right)(x)-p(x) \\
\leq & -k\left(W^{\mathrm{T}} A^{-1} W\right)(x)-\left(B^{\mathrm{T}} A^{-1} W\right)(x)-p(x) \\
\leq & -\frac{k}{\lambda_{\max }(x)}\left(W^{\mathrm{T}} W\right)(x)-\left(B^{\mathrm{T}} A^{-1} W\right)(x)-p(x) \\
\leq & -\frac{k}{2 \lambda_{\max }(x)}\left(W^{\mathrm{T}} W\right)(x)+\frac{1}{2 k} \lambda_{\max }(x)\left|B^{\mathrm{T}} A^{-1}\right|^{2}-p(x),
\end{aligned}
$$

where the last inequality follows by Lemma 2.1. Using the divergence theorem in (2.2), by (2.4), we obtain

$$
\begin{aligned}
Z^{\prime}(r) & =\frac{\varrho^{\prime}(r)}{\varrho(r)} Z(r)+\varrho(r)\left\{\int_{S_{r}} \operatorname{div} W(x) d \sigma+[\lambda(r) \eta(r)]^{\prime}\right\} \\
& \leq \frac{\varrho^{\prime}(r)}{\varrho(r)} Z(r)-\varrho(r)\left\{\frac{k}{2 \lambda(r)} \int_{S_{r}}\left(W^{\mathrm{T}} W\right)(x) d \sigma+p_{M}(r)-[\lambda(r) \eta(r)]^{\prime}\right\} .
\end{aligned}
$$

By the Schwarz inequality,

$$
\int_{S_{r}}|W(x)|^{2} d \sigma \geq \frac{r^{1-N}}{\omega_{N}}\left[\int_{S_{r}} W(x) \cdot \nu(x) d \sigma\right]^{2} .
$$

Therefore,

$$
\begin{aligned}
Z^{\prime}(r) \leq & \frac{\varrho^{\prime}(r)}{\varrho(r)} Z(r) \\
& -\varrho(r)\left\{\frac{k}{2 \omega_{N}} \frac{r^{1-N}}{\lambda(r)}\left(\int_{S_{r}} W(x) \cdot \nu(x) d \sigma\right)^{2}+p_{M}(r)-[\lambda(r) \eta(r)]^{\prime}\right\}
\end{aligned}
$$




$$
\begin{aligned}
= & \frac{\varrho^{\prime}(r)}{\varrho(r)} Z(r) \\
& -\varrho(r)\left\{\frac{k}{2 \omega_{N}} \frac{r^{1-N}}{\lambda(r)}\left(\frac{Z(r)}{\varrho(r)}-\lambda(r) \eta(r)\right)^{2}+p_{M}(r)-[\lambda(r) \eta(r)]^{\prime}\right\} \\
= & -\theta(r)-\frac{1}{g(r)} Z^{2}(r),
\end{aligned}
$$

that is, $Z(r)$ satisfies the Riccati inequality (2.3).

THEOREM 2.1. Suppose that there exist functions $\eta \in C^{1}([a, \infty), \mathbb{R})$ and $\phi \in \Im$ such that

$$
\int_{b}^{\infty} \frac{\alpha^{\mu}(s, b)}{\beta(s, b)} d s=\infty \quad \text { for some } b \geq a, \text { and } 0 \leq \mu<1,
$$

and

$$
\lim _{r \rightarrow \infty} \Psi_{\phi}(r, a)=\infty
$$

Then equation (1.1) is oscillatory.

Proof. Suppose $y=y(x)$ is a nonoscillatory solution of (1.1). We may assume that there exists a $b_{0} \geq a$ such that $y(x)>0$ for $x \in \Omega\left(b_{0}\right)$. A similar argument holds for $y(x)<0$. For $x \in \Omega\left(b_{0}\right)$, define $Z(r)$ by $(2.2)$. In view of Lemma 2.2, we know that (2.3) holds. It follows that

$$
Z(r)+\int_{b}^{r} \frac{1}{g(s)} Z^{2}(s) d s \leq L_{1}-\int_{b}^{r} \theta(s) d s \quad \text { for } r \geq b \geq b_{0},
$$

where $L_{1}$ is a constant. Multiplying $(2.7)$ by $\phi(s)$ and integrating from $b$ to $r$, we get

$$
\int_{b}^{r} \phi(s) Z(s) d s+\int_{b}^{r} \phi(s) \int_{b}^{s} \frac{1}{g(u)} Z^{2}(u) d u d s \leq \alpha(r, b)\left[L_{1}-\Psi_{\phi}(r, b)\right] .
$$

Note that by (2.6), there exists a $b_{1}>b$ such that $L_{1}-\Psi_{\phi}(r, b)<0$ for all $r \geq b_{1}$. Then, for any $r \geq b_{1}$,

$$
G(r):=\int_{b}^{r} \phi(s) \int_{b}^{s} \frac{1}{g(u)} Z^{2}(u) d u d s \leq-\int_{b}^{r} \phi(s) Z(s) d s .
$$

Since $G(r)$ is nonnegative, by Schwarz's inequality we have

$$
G^{2}(r) \leq\left(\int_{b}^{r} \phi(s) Z(s) d s\right)^{2} \leq\left(\int_{b}^{r} g(s) \phi^{2}(s) d s\right)\left(\int_{b}^{r} \frac{1}{g(s)} Z^{2}(s) d s\right) .
$$


On the other hand,

$$
G(r) \geq \int_{b}^{r} \phi(s) \int_{b}^{b_{1}} \frac{1}{g(s)} Z^{2}(s) d s=L_{2} \alpha(r, b)
$$

where $L_{2}=\int_{b}^{b_{1}}\left(Z^{2}(s) / g(s)\right) d s$. From (2.8) and (2.9), we obtain

$$
L_{2}^{\mu} \frac{\alpha^{\mu}(r, b)}{\beta(r, b)} \leq G^{\mu-2}(r) G^{\prime}(r)
$$

for all $r \geq b_{1}$ and $0 \leq \mu<1$. Integrating (2.10) from $b_{1}$ to $r$ yields

$$
L_{2}^{\mu} \int_{b_{1}}^{r} \frac{\alpha^{\mu}(s, b)}{\beta(s, b)} d s \leq \frac{1}{1-\mu} \frac{1}{G^{1-\mu}\left(b_{1}\right)}<\infty
$$

which contradicts (2.5). Thus, we conclude that (1.1) is oscillatory.

The following theorem can be used when Theorem 2.1 cannot be applied.

TheOREM 2.2. Suppose that there exist functions $\eta \in C^{1}([a, \infty), \mathbb{R})$ and $\phi \in C^{1}\left([a, \infty), \mathbb{R}^{+}\right)$such that

$$
\begin{gathered}
\int_{a}^{\infty} \frac{g(s)}{\phi(s)} \phi^{2}(s) d s<\infty, \\
\int_{a}^{\infty} \phi(s) \theta(s) d s=\infty, \\
\int_{a}^{\infty} \frac{d s}{g(s) \phi(s)}=\infty .
\end{gathered}
$$

Then equation (1.1) is oscillatory.

Proof. Suppose to the contrary that there exist a solution $y=y(x)$ of (1.1) and a constant $b_{0} \geq a$ such that $y(x)>0$ for $x \in \Omega\left(b_{0}\right)$. By Lemma $2.2,(2.3)$ holds for $r \geq b_{0}$. Multiplying (2.3) by $\phi(s)$, and integrating both sides of the resulting inequality over $[b, r], b \geq b_{0}$, we obtain

$$
\phi(r) Z(r) \leq L_{3}+\int_{b}^{r} \phi^{\prime}(s) Z(s) d s-\int_{b}^{r} \frac{\phi(s)}{g(s)} Z^{2}(s) d s-\int_{b}^{r} \phi(s) \theta(s) d s
$$

where $L_{3}$ is a constant. Schwarz's inequality yields

$$
\begin{aligned}
\int_{b}^{r}\left|\phi^{\prime}(s) Z(s)\right| d s & \leq\left(\int_{b}^{r} \frac{g(s)}{\phi(s)} \phi^{\prime 2}(s) d s\right)^{1 / 2}\left(\int_{b}^{r} \frac{\phi(s)}{g(s)} Z^{2}(s) d s\right)^{1 / 2} \\
& \leq L_{4}\left(\int_{b}^{r} \frac{\phi(s)}{g(s)} Z^{2}(s) d s\right)^{1 / 2}=: L_{4} T^{1 / 2}(r),
\end{aligned}
$$


where $L_{4}=\left(\int_{b}^{\infty} g(s)\left(\phi^{\prime 2}(s) / \phi(s)\right) d s\right)^{1 / 2}$ is finite by (2.11) and the function $T(r)$ is defined by the last equality. Hence, (2.14) implies that

$$
\phi(r) Z(r) \leq L_{3}+L_{4} T^{1 / 2}(r)-T(r)-\int_{b}^{r} \phi(s) \theta(s) d s
$$

for every $r \geq b$. Since the function $L_{4} T^{1 / 2}(r)-T(r) / 2$ is bounded from above on $\mathbb{R}^{+}$, assumption (2.12) shows that the right hand side of (2.15) tends to $-\infty$ as $r \rightarrow \infty$. So, we can find a constant $b_{1} \geq b_{0}$ such that for all $r \geq b_{1}$,

$$
\phi(r) Z(r) \leq-\frac{1}{2} T(r) .
$$

Thus,

$$
T^{\prime}(r)=\frac{\phi(r)}{g(r)} Z^{2}(r) \geq \frac{1}{4} \frac{1}{\phi(r) g(r)} T^{2}(r) .
$$

Dividing both sides of the above inequality by $T^{2}(r)$ and integrating from $b_{1}$ to $r$, we have

$$
\frac{1}{T\left(b_{1}\right)} \geq \frac{1}{4} \int_{b_{1}}^{r} \frac{d s}{\phi(s) g(s)},
$$

which contradicts (2.13). Hence, (1.1) is oscillatory.

Remark 2.1. Putting $\phi(r)=1$ in Theorem 2.2, one improves Theorem 4 in [13] for (1.2).

REMARK 2.2. Theorems 2.1 and 2.2 are quite general; some interesting corollaries can be drawn by choosing particular functions $\varrho$ and $\phi$. The details are left to the interested reader.

3. Kamenev type criteria. In this section, we will establish analogues of Kamenev type [6], and more generally, Philos type [15] criteria for oscillation of (1.1). For this reason, we introduce a class $\mathcal{H}$ of functions. Let

$$
D=\{(r, s): r \geq s \geq a\}, \quad D_{0}=\{(r, s): r>s \geq a\} .
$$

A function $H=H(r, s) \in C(D, \mathbb{R})$ is said to belong to a function class $\mathcal{H}$ if

(H1) $H(r, r)=0$ for $r \geq a, H(r, s)>0$ on $D_{0}$;

(H2) $H$ has a continuous and nonpositive partial derivative on $D_{0}$ with respect to the second variable, and there exists a function $h \in$ $C\left(D_{0}, \mathbb{R}\right)$ such that

$$
-\frac{\partial H}{\partial s}(r, s)=h(r, s) H(r, s) \quad \text { for all }(r, s) \in D_{0} .
$$


Given $H \in \mathcal{H}$ and $\kappa \in C\left(D_{0}, \mathbb{R}\right)$, we define an integral operator $\Pi_{a}^{r}$ as

$$
\Pi_{a}^{r}(\kappa)=\frac{1}{H(r, a)} \int_{a}^{r} H(r, s) \kappa(r, s) d s \quad \text { for } r>a .
$$

TheOREm 3.1. Suppose that there exist functions $\eta \in C^{1}\left([a, \infty), \mathbb{R}^{+}\right)$ and $H \in \mathcal{H}$ such that

$$
\limsup _{r \rightarrow \infty} \Pi_{a}^{r}\left(\theta-\frac{1}{4} g h^{2}\right)=\infty .
$$

Then equation (1.1) is oscillatory.

Proof. Suppose $y=y(x)$ is a nonoscillatory solution of (1.1). We may assume that there exists a $b_{0} \geq a$ such that $y(x)>0$ for $x \in \Omega\left(b_{0}\right)$. By Lemma $2.2,(2.3)$ holds for $r \geq b_{0}$. Multiplying $(2.3)$ by $H(r, s)$ and integrating from $b\left(b \geq b_{0}\right)$ to $r$, by (H2), leads to

$$
\begin{aligned}
\Pi_{b}^{r}(\theta) & \leq Z(b)-\Pi_{b}^{r}(h Z)-\Pi_{b}^{r}\left(g^{-1} Z^{2}\right) \\
& =Z(b)+\frac{1}{4} \Pi_{b}^{r}\left(g h^{2}\right)-\Pi_{b}^{r}\left(g^{-1}\left(Z+\frac{1}{2} g h\right)^{2}\right) .
\end{aligned}
$$

Thus,

$$
\Pi_{b}^{r}\left(\theta-\frac{1}{4} g h^{2}\right) \leq Z(b) .
$$

Moreover, by (3.2) and (H2), for all $r \geq a$, we obtain

$$
\begin{aligned}
H(r, a) \Pi_{a}^{r}\left(\theta-\frac{1}{4} g h^{2}\right)= & H\left(b_{0}, a\right) \Pi_{a}^{b_{0}}\left(\theta-\frac{1}{4} g h^{2}\right) \\
& +H\left(r, b_{0}\right) \Pi_{b_{0}}^{r}\left(\theta-\frac{1}{4} g h^{2}\right) \\
\leq & H(r, a)\left[\int_{a}^{b_{0}}|\theta(s)| d s+\left|Z\left(b_{0}\right)\right|\right] .
\end{aligned}
$$

This gives

$$
\limsup _{r \rightarrow \infty} \Pi_{a}^{r}\left(\theta-\frac{1}{4} g h^{2}\right) \leq \int_{a}^{b_{0}}|\theta(s)| d s+|Z(a)|,
$$

which contradicts (3.1). The proof is now complete.

From Theorem 3.1 the following corollary is immediate.

Corollary 3.1. Let the assumptions of Theorem 3.1 hold with (3.1) replaced by

$$
\limsup _{r \rightarrow \infty} \Pi_{a}^{r}(\theta)=\infty
$$


and

$$
\limsup _{r \rightarrow \infty} \Pi_{a}^{r}\left(g h^{2}\right)<\infty
$$

Then equation (1.1) is oscillatory.

It is clear that (3.3) is a necessary condition for (3.1) to hold. In case (3.3) fails, the following theorem may be applicable.

THEOREM 3.2. Suppose that there exist functions $\eta \in C^{1}\left([a, \infty), \mathbb{R}^{+}\right)$ and $H \in \mathcal{H}$ such that

$$
0<\inf _{s \geq a}\left\{\liminf _{r \rightarrow \infty} \frac{H(r, s)}{H(r, a)}\right\} \leq \infty
$$

and

$$
\limsup _{r \rightarrow \infty} \Pi_{a}^{r}\left(g h^{2}\right)<\infty
$$

Let $\psi \in C([a, \infty), \mathbb{R})$ be such that

$$
\int_{a}^{\infty} \frac{\psi_{+}^{2}(s)}{g(s)} d s=\infty
$$

and that for all $b \geq a$,

$$
\limsup _{r \rightarrow \infty} \Pi_{b}^{r}\left(\theta-\frac{1}{4} g h^{2}\right) \geq \psi(b),
$$

where $\psi_{+}=\max \{\psi, 0\}$. Then equation (1.1) is oscillatory.

Proof. Proceeding as in the proof of Theorem 3.1, we see that (3.2) holds. Furthermore, for $r \geq b \geq b_{0}$,

$$
\Pi_{b}^{r}\left(\theta-\frac{1}{4} g h^{2}\right) \leq Z(b)-\Pi_{b}^{r}\left(g^{-1}\left(Z+\frac{1}{2} g h\right)^{2}\right) .
$$

Hence, for $b \geq b_{0}$,

$$
\limsup _{r \rightarrow \infty} \Pi_{b}^{r}\left(\theta-\frac{1}{4} g h^{2}\right) \leq Z(b)-\liminf _{r \rightarrow \infty} \Pi_{b}^{r}\left(g^{-1}\left(Z+\frac{1}{2} g h\right)^{2}\right) .
$$

By (3.8), we have

$$
Z(b) \geq \psi(b)+\liminf _{r \rightarrow \infty} \Pi_{b}^{r}\left(g^{-1}\left(Z+\frac{1}{2} g h\right)^{2}\right) .
$$

So, for all $b \geq a$,

$$
Z(b) \geq \psi(b) .
$$

By (3.9), we get

$$
\liminf _{r \rightarrow \infty} \Pi_{b_{0}}^{r}\left(g^{-1}\left(Z+\frac{1}{2} g h\right)^{2}\right) \leq Z\left(b_{0}\right)-\psi\left(b_{0}\right)<\infty,
$$


and thus

$$
\begin{aligned}
\liminf _{r \rightarrow \infty}\left\{\Pi_{b_{0}}^{r}\left(g^{-1} Z^{2}\right)+\right. & \left.\Pi_{b_{0}}^{r}(h Z)\right\} \\
& \leq \liminf _{r \rightarrow \infty} \Pi_{b_{0}}^{r}\left(g^{-1}\left(Z+\frac{1}{2} g h\right)^{2}\right)<\infty
\end{aligned}
$$

Now, we claim that

$$
\int_{b_{0}}^{\infty} \frac{Z^{2}(s)}{g(s)} d s<\infty .
$$

Suppose to the contrary that

$$
\int_{b_{0}}^{\infty} \frac{Z^{2}(s)}{g(s)} d s=\infty
$$

By (3.5), there exists a positive constant $\xi$ such that

$$
\inf _{s \geq a}\left\{\liminf _{r \rightarrow \infty} \frac{H(r, s)}{H(r, a)}\right\}>\xi>0 .
$$

On the other hand, by (3.13) for any positive number $\gamma$ there exists $b_{1}>b_{0}$ such that

$$
\int_{b_{0}}^{r} \frac{Z^{2}(s)}{g(s)} d s \geq \frac{\gamma}{\xi} \quad \text { for all } r \geq b_{1}
$$

so, for all $r \geq b_{1}$,

$$
\begin{aligned}
\Pi_{b_{0}}^{r}\left(g^{-1} Z^{2}\right) & =\frac{1}{H\left(r, b_{0}\right)} \int_{b_{0}}^{r} H(r, s) d\left(\int_{b_{0}}^{s} \frac{1}{g(u)} Z^{2}(u) d u\right) \\
& =\frac{1}{H\left(r, b_{0}\right)} \int_{b_{0}}^{r}\left(-\frac{\partial H}{\partial s}(r, s)\right)\left(\int_{b_{0}}^{s} \frac{1}{g(u)} Z^{2}(u) d u\right) d s \\
& \geq \frac{1}{H\left(r, b_{0}\right)} \int_{b_{1}}^{r}\left(-\frac{\partial H}{\partial s}(r, s)\right)\left(\int_{b_{0}}^{r} \frac{1}{g(u)} Z^{2}(u) d u\right) d s \\
& \geq \frac{\gamma}{\xi H\left(r, b_{0}\right)} \int_{b_{1}}^{r}\left(-\frac{\partial H}{\partial s}(r, s)\right) d s=\frac{\gamma H\left(r, b_{1}\right)}{\xi H\left(r, b_{0}\right)} .
\end{aligned}
$$

From (3.14), we have

$$
\liminf _{r \rightarrow \infty} \frac{H\left(r, b_{1}\right)}{H(r, a)}>\xi>0 .
$$

Hence, there exists $b_{2}>b_{1}$ such that $H\left(r, b_{1}\right) / H(r, a)>\xi$ for all $r \geq b_{2}$. Therefore, by $(3.15), \Pi_{b_{0}}^{r}\left(g^{-1} Z^{2}\right)>\gamma$ for all $r \geq b_{2}$, and since $\gamma$ is an arbitrary constant, we conclude that

$$
\lim _{r \rightarrow \infty} \Pi_{b_{0}}^{r}\left(g^{-1} Z^{2}\right)=\infty
$$


Next, consider a sequence $\left\{r_{n}\right\}_{n=1}^{\infty}$ in $\left(b_{0}, \infty\right)$ such that $\lim _{n \rightarrow \infty} r_{n}=\infty$ and

$$
\lim _{n \rightarrow \infty}\left\{\Pi_{b_{0}}^{r_{n}}\left(g^{-1} Z^{2}\right)+\Pi_{b_{0}}^{r_{n}}(h Z)\right\}=\liminf _{r \rightarrow \infty}\left\{\Pi_{b_{0}}^{r}\left(g^{-1} Z^{2}\right)+\Pi_{b_{0}}^{r}(h Z)\right\} .
$$

In view of (3.11), there exists a constant $M$ such that

$$
\Pi_{b_{0}}^{r_{n}}\left(g^{-1} Z^{2}\right)+\Pi_{b_{0}}^{r_{n}}(h Z) \leq M \quad \text { for } n=1,2, \ldots
$$

It follows from (3.16) that

$$
\lim _{n \rightarrow \infty} \Pi_{b_{0}}^{r_{n}}\left(g^{-1} Z^{2}\right)=\infty
$$

This and (3.17) yield

$$
\lim _{n \rightarrow \infty} \Pi_{b_{0}}^{r_{n}}(h Z)=-\infty
$$

Then, by (3.17) and (3.18),

$$
1+\frac{\Pi_{b_{0}}^{r_{n}}(h Z)}{\Pi_{b_{0}}^{r_{n}}\left(g^{-1} Z^{2}\right)} \leq \frac{M}{\Pi_{b_{0}}^{r_{n}}\left(g^{-1} Z^{2}\right)}<\frac{1}{2} \quad \text { for large enough }
$$

and consequently,

$$
\frac{\Pi_{b_{0}}^{r_{n}}(h Z)}{\Pi_{b_{0}}^{r_{n}}\left(g^{-1} Z^{2}\right)}<-\frac{1}{2} \quad \text { for } n \text { large enough. }
$$

This and (3.19) imply that

$$
\lim _{n \rightarrow \infty} \frac{\left[\Pi_{b_{0}}^{r_{n}}(h Z)\right]^{2}}{\Pi_{b_{0}}^{r_{n}}\left(g^{-1} Z^{2}\right)}=\infty
$$

Furthermore, by the Schwarz inequality, for any natural number $n$ we have

$$
\frac{\left[\Pi_{b_{0}}^{r_{n}}(h Z)\right]^{2}}{\Pi_{b_{0}}^{r_{n}}\left(g^{-1} Z^{2}\right)} \leq \Pi_{b_{0}}^{r_{n}}\left(g h^{2}\right) \leq \Pi_{a}^{r_{n}}\left(g h^{2}\right)
$$

It follows from (3.20) and (3.21) that

$$
\lim _{n \rightarrow \infty} \Pi_{a}^{r_{n}}\left(g h^{2}\right)=\infty
$$

and this implies

$$
\limsup _{r \rightarrow \infty} \Pi_{a}^{r}\left(g h^{2}\right)=\infty
$$

which contradicts assumption (3.6). Therefore we have proved that (3.13) fails, so (3.12) holds true. Then it follows from (3.10) that $\psi_{+}^{2}(b) \leq Z^{2}(b)$ for all $b \geq b_{0}$, hence

$$
\int_{b_{0}}^{\infty} \frac{\psi_{+}^{2}(s)}{g(s)} d s \leq \int_{b_{0}}^{\infty} \frac{1}{g(s)} Z^{2}(s) d s<\infty
$$

which contradicts condition (3.7). This completes the proof. 
Using the same techniques, we may obtain two slightly different forms of Theorem 3.2, which we state here for completeness.

ThEOREM 3.3. Suppose that there exist functions $\eta \in C^{1}\left([a, \infty), \mathbb{R}^{+}\right)$ and $H \in \mathcal{H}$ such that (3.5) holds, and

$$
\liminf _{r \rightarrow \infty} \Pi_{a}^{r}(\theta)<\infty .
$$

Let $\psi \in C([a, \infty), \mathbb{R})$ be such that $(3.7)$ holds and for all $b \geq a$,

$$
\liminf _{r \rightarrow \infty} \Pi_{b}^{r}\left(\theta-\frac{1}{4} g h^{2}\right) \geq \psi(b) .
$$

Then equation (1.1) is oscillatory.

ThEOREM 3.4. Suppose that there exist functions $\eta \in C^{1}\left([a, \infty), \mathbb{R}^{+}\right)$ and $H \in \mathcal{H}$ such that (3.5) holds, and

$$
\liminf _{r \rightarrow \infty} \Pi_{a}^{r}\left(g h^{2}\right)<\infty
$$

Let $\psi \in C([a, \infty), \mathbb{R})$ be such that (3.7) and (3.23) hold. Then equation (1.1) is oscillatory.

REMARK 3.1. For (1.2), Theorems 3.1-3.4 reduce to Theorems 2.1-2.4 in [19].

REMARK 3.2. From Theorems 3.1-3.4, by an appropriate choice of the function $H$, we can deduce several different explicit sufficient conditions for the oscillation of (1.1). For instance, choosing

$$
H(r, s)=(r-s)^{m},[G(r)-G(s)]^{m}, \log \left[\frac{Q(r)}{Q(s)}\right]^{m}, \text { or }\left[\int_{s}^{r} \frac{d z}{u(z)}\right]^{m}
$$

for $r \geq s \geq a$, where $m>1$ is a constant, and

$$
G(r)=\int_{a}^{r} \frac{d s}{g(s)}, \quad Q(r)=\int_{r}^{\infty} \frac{d s}{g(s)}<\infty
$$

for $r \geq a$, and $u \in C\left([a, \infty), \mathbb{R}^{+}\right)$satisfying $\int_{a}^{\infty} d z / u(z)=\infty$, we can derive some effective oscillation criteria for (1.1).

REMARK 3.3. The results obtained in this paper hold true if we replace condition (A3) with the following one:

$\left(\mathrm{A} 3^{\prime}\right) f \in C(\mathbb{R}, \mathbb{R}), f(y) / y>k>0$ for $y \neq 0$.

But the function $p$ should be nonnegative in this case.

4. Examples. In the last section, we give three examples to illustrate our main results. As far as the author knows, no previously known criteria for oscillation can be applied to these examples. 
EXAmple 4.1. Consider equation (1.1) with

$$
\begin{aligned}
A & =\operatorname{diag}\left(\frac{1}{|x|^{2}}, \frac{1}{|x|^{2}}\right), \quad B=\left(\frac{\sin |x|}{|x|^{9 / 4}}, \frac{\cos |x|}{|x|^{9 / 4}}\right), \\
p(x) & =\frac{4+\cos |x|-2|x| \sin |x|}{4|x|^{5 / 2}}, \quad f(y)=y+y^{3},
\end{aligned}
$$

where $N=2$ and $x \in \Omega(1)$. Let $\eta(r)=-2 \pi$ and $\phi(r)=1 / r$. A direct calculation implies that

$$
\begin{aligned}
\varrho(r) & =r, \quad g(r)=4 \pi, \quad \theta(r)=\frac{\pi(2+\cos r-2 r \sin r)}{2 r^{3 / 2}}-\frac{3 \pi}{r^{2}}, \\
\alpha(r, 1) & =\ln r, \quad \beta(r, a)=4 \pi(r-1) .
\end{aligned}
$$

Now, we can easily show that

$$
\begin{aligned}
\int_{1}^{r} \theta(s) d s & =\pi[\sqrt{r}(2+\cos r)-(2+\cos 1)]+3 \pi\left(\frac{1}{r}-1\right) \\
& \geq \pi\left[\sqrt{r}-C_{1}\right], \quad C_{1} \text { constant, } \\
\Psi_{\phi}(r, 1) & \geq \frac{\pi}{\ln r} \int_{1}^{r} \frac{1}{s}\left(\sqrt{s}-C_{1}\right) d s \\
& =\frac{\pi}{\ln r} \int_{1}^{r}\left(\frac{1}{\sqrt{s}}-\frac{C_{1}}{s}\right) d s \rightarrow \infty \quad \text { as } r \rightarrow \infty,
\end{aligned}
$$

and

$$
\int_{1}^{r} \frac{\alpha^{\mu}(s, 1)}{\beta(s, 1)} d s=\frac{1}{4 \pi} \int_{1}^{s} \frac{\ln ^{\mu} s}{s-1} d s \rightarrow \infty \quad \text { as } r \rightarrow \infty .
$$

Thus, all conditions of Theorem 2.1 are satisfied, and hence equation (4.1) is oscillatory.

Example 4.2. Consider equation (1.1) with

$$
\begin{aligned}
A & =\operatorname{diag}(1,1), \quad B=\left(\frac{1}{|x|^{2}}, \frac{1}{|x|^{2}}\right), \\
p(x) & =\tau|x|^{\tau-2}(2-\cos |x|)+|x|^{\tau-1} \sin |x|, \quad f(y)=y+y^{3},
\end{aligned}
$$

where $N=2, x \in \Omega(1)$ and $\tau>0$. Let $\eta(r)=0$. By a direct calculation,

$$
\varrho(r)=1, \quad g(r)=4 \pi r, \quad \theta(r)=2 \pi\left[\tau r^{\tau-1}(2-\cos r)+r^{\tau} \sin r-\frac{1}{r^{3}}\right] .
$$

Hence, for $r \geq 1$, we get 


$$
\begin{aligned}
\int_{1}^{r} \theta(s) d s & =2 \pi \int_{1}^{r} d\left(s^{\tau}(2-\cos s)+\frac{1}{2 s^{2}}\right) \\
& =2 \pi\left[r^{\tau}(2-\cos r)+\frac{1}{2 r^{2}}-(3-\cos 1)\right] \\
& \geq r^{\tau}-C_{2}, \quad \text { with } C_{2} \text { a constant. }
\end{aligned}
$$

For Theorem 3.1, taking $H(r, s)=(r-s)^{2}$, we obtain

$$
\begin{aligned}
\Pi_{1}^{r}\left(\theta-\frac{1}{4} g h^{2}\right) & =\frac{1}{r^{2}} \int_{1}^{r}(r-s)^{2}\left(\theta(s)-\frac{1}{4} g(s) h^{2}(r, s)\right) d s \\
& =\frac{1}{r^{2}} \int_{1}^{r}\left(2(r-s) \int_{1}^{s} \theta(u) d u-4 \pi s\right) d s \\
& \geq \frac{2}{r^{2}} \int_{1}^{r}(r-s)\left(s^{\tau}-C_{2}\right) d s-2 \pi\left(1-\frac{1}{r^{2}}\right) \rightarrow \infty
\end{aligned}
$$

as $r \rightarrow \infty$. Consequently, condition (3.1) is satisfied. Hence (4.2) is oscillatory by Theorem 3.1.

ExAmPle 4.3. Consider equation (1.1) with

$$
\begin{gathered}
A=\operatorname{diag}\left(\frac{1}{|x|^{2}}, \frac{1}{|x|^{2}}\right), \quad B=(1,1), \\
p(x)=|x|^{\tau-1} \cos |x|, \quad f(y)=y+y^{3},
\end{gathered}
$$

where $N=2, x \in \Omega(1)$ and $\tau \geq-1$. Let $\eta(r)=2 \pi$. A straightforward calculation yields

$$
\varrho(r)=\frac{1}{r}, \quad g(r)=\frac{4 \pi}{r}, \quad \theta(r)=\pi\left(2 r^{\tau} \cos r+\frac{3}{r^{5}}\right) .
$$

Furthermore, for simplicity, let $H(r, s)=(r-s)^{2}$; then

$$
\limsup _{r \rightarrow \infty} \frac{1}{r^{2}} \Pi_{1}^{r}\left(g h^{2}\right)=16 \pi \limsup _{r \rightarrow \infty} \frac{1}{r^{2}} \int_{1}^{r} \frac{1}{s} d s=0,
$$

so (3.6) holds. For any $b \geq 1$,

$$
\begin{aligned}
\limsup _{r \rightarrow \infty} \Pi_{b}^{r}( & \left.\theta-\frac{1}{4} g h^{2}\right) \\
& =\pi \limsup _{r \rightarrow \infty} \frac{1}{r^{2}} \int_{b}^{r}(r-s)^{2}\left(2 s^{\tau} \cos s+\frac{3}{s^{5}}-\frac{16}{s(r-s)^{2}}\right) d s \\
& \geq-\pi b^{\tau} \sin b-C_{3}
\end{aligned}
$$


where $C_{3}$ is a positive constant. Now let $\psi(b)=-\pi b^{\tau} \sin b-C_{3}$. Then there exists an integer $J$ such that $(2 n J+1) \pi+\pi / 4>1$ and, for all $n \geq J$,

$$
\psi(b) \geq-\pi b^{\tau} \sin b-C_{3} \geq \epsilon b^{\tau} \quad \text { for } b \in\left[(2 n+1) \pi+\frac{\pi}{4}, 2(n+1) \pi-\frac{\pi}{4}\right],
$$

where $\epsilon$ is a small constant. Thus, taking into account that $\tau>-1$, we get

$$
\begin{aligned}
\lim _{r \rightarrow \infty} \int_{1}^{r} \frac{\psi_{+}^{2}(s)}{g(s)} d s & =\frac{1}{4 \pi} \lim _{r \rightarrow \infty} \int_{1}^{r} s \psi_{+}^{2}(s) d s \geq \frac{\epsilon^{2}}{4 \pi} \sum_{n=J}^{\infty} \int_{(2 n+1) \pi+\pi / 4}^{2(n+1) \pi-\pi / 4} s^{2 \tau+1} d s \\
& \geq \frac{\epsilon^{2}}{4 \pi} \sum_{n=J}^{\infty} \int_{(2 n+1) \pi+\pi / 4}^{2(n+1) \pi-\pi / 4} s^{-1} d s=\infty,
\end{aligned}
$$

so condition (3.7) is also satisfied. Thus (4.3) is oscillatory by Theorem 3.2.

Acknowledgments. The author is grateful to the referee for his/her valuable comments and suggestions which have led to an improvement of the presentation.

\section{References}

[1] W. Allegretto, Oscillation criteria for quasilinear equations, Canad. J. Math. 26 (1974), 931-947.

[2] - Oscillation criteria for semilinear equations in general domains, Canad. Math. Bull. 19 (1976), 137-144.

[3] W. J. Coles, An oscillation criterion for second-order linear differential equations, Proc. Amer. Math. Soc. 19 (1968), 755-759.

[4] W. B. Fite, Concerning the zeros of the solutions of certain differential equations, Trans. Amer. Math. Soc. 19 (1918), 341-352.

[5] D. Gilbarg and N. S. Trudinger, Elliptic Partial Differential Equations of Second Order, Springer, New York, 1983.

[6] I. V. Kamenev, An integral criterion for oscillation of linear differential equations of second order, Mat. Zametki 23 (1978), 249-251 (in Russian); English transl.: Math. Notes 23 (1978), 136-138.

[7] Y. Kitamura and T. Kusano, An oscillation theorem for a sublinear Schrödinger equation, Utilitas Math. 14 (1978), 171-175.

[8] T. Kura, Oscillation criteria for a class of sublinear elliptic equations of the second order, ibid. 22 (1982), 335-341.

[9] T. Kusano and M. Naito, Oscillation theory of entire solutions of second order superlinear elliptic equations, Funkcial. Ekvac. 30 (1987), 269-282.

[10] M. Naito, Y. Naito and H. Usami, Oscillation theory for semilinear elliptic equations with arbitrary nonlinearities, ibid. 40 (1997), 41-55.

[11] M. Naito and N. Yoshida, Oscillation theorems for semilinear elliptic differential operators, Proc. Roy. Soc. Edinburgh Sect. A 82 (1978), 135-151.

[12] Y. Naito and H. Usami, Oscillation criteria for quasilinear elliptic equations, Nonlinear Anal. 46 (2001), 629-652. 
[13] E. S. Noussair and C. A. Swanson, Oscillation of semilinear elliptic inequalities by Riccati transformation, Canad. J. Math. 32 (1980), 908-923.

[14] - - - Oscillation theory for semilinear Schrödinger equations and inequalities, Proc. Roy. Soc. Edinburgh Sect. A 75 (1975/76), 67-81.

[15] Ch. G. Philos, Oscillation theorems for linear differential equations of second order, Arch. Math. (Basel) 53 (1989), 482-492.

[16] C. A. Swanson, Criteria for oscillatory sublinear Schrödinger equations, Pacific J. Math. 104 (1983), 483-493.

[17] —, Semilinear second order elliptic oscillation, Canad. Math. Bull. 22 (1979), 139157.

[18] Z. T. Xu, Oscillation of solutions to second order elliptic partial differential equations with a "weakly integrally small" coefficient, J. Systems Sci. Math. Sci. 18 (1998), 478-484 (in Chinese).

[19] -, Riccati techniques and oscillation of semilinear elliptic equations, Chinese J. Contemp. Math. 24 (2003), 329-340.

[20] Z. T. Xu, D. K. Ma and B. G. Jia, Oscillation theorems for elliptic equations of second order, Acta Math. Sci. Ser. A 24 (2004), 144-151 (in Chinese).

[21] N. Yoshida, Oscillation properties of solutions of second order elliptic equations, SIAM J. Math. Anal. 14 (1983), 709-718.

[22] B. G. Zhang, T. Zhao and B. S. Lalli, Oscillation criteria for nonlinear second order elliptic differential equations, Chinese Ann. Math. 17 (1996), 89-102.

School of Mathematical Sciences

South China Normal University

Guangzhou, 510090, P.R. China

E-mail: xztxhyyj@pub.guangzhou.gd.cn

Received 28.7.2005

and in final form 10.2.2007 